\title{
GlobalBioIm: A Unifying Computational Framework for Solving Inverse Problems
}

\author{
Michael Unser, Emmanuel Soubies, Ferréol Soulez, Michael McCann, Laurène Donati \\ Biomedical Imaging Group, École polytechnique fédérale de Lausanne (EPFL), CH-1015 Lausanne, Switzerland. \\ michael.unser@epfl.ch
}

\begin{abstract}
We present a unifying framework for the development of state-of-the-art reconstruction algorithms in computational optics with a clear separation between the physical (forward model) and signal-related (regularization, incorporation of prior constraints) aspects of the problem. The pillars of our formulation are: (i) an operator algebra with its set of fast linear solvers, (ii) a variational derivation of reconstruction methods, and (iii) a suite of efficient numerical tools for the resolution of large-scale optimization problems. These core technologies are incorporated into a modular software library featuring the key components for the implementation and testing of iterative reconstruction algorithms. The concept is illustrated with concrete examples in 3D deconvolution microscopy and lenseless imaging.
\end{abstract}

OCIS codes: $100.3190,110.1758,110.3010,110.6955$

\section{Introduction}

A major trend in optical imaging is the shift towards computational imaging where sophisticated algorithms are being deployed in order to reconstruct the information of interest from optical measurements. These measurements can take the form of traditional images, as in the case of 3D deconvolution microscopy [1], interferograms/holograms, modulations [2], or 2D projections of a specimen as in refractive-index tomography [3].

While the combination of optics and advanced signal processing offers unprecedented opportunities, it also comes with its own difficulties. When being confronted with a new imaging problem, the common experience is that one has to reimplement (if not reinvent) the wheel (=forward model + optimization algorithm), which is very time consuming and also acts as a deterrent for engaging in new developments. The present framework (GlobalBioIm) aims at simplifying this process by decomposing the workflow onto smaller modules, including many reusable ones since several aspects such as regularization and the injection of prior knowledge are rather generic. It also capitalizes on the strong commonalities between the various image formation models that can be exploited to obtain fast, streamlined implementations. We are planning to make GlobalBioIm available as a documented resource for algorithm developers and researchers in optics to test new ideas and compare their methods against the state of the art.

The premise of GlobalBioIm is that it is possible-in keeping with the very successful ADMM philosophyto solve complex problems by decomposing them into a sequence of simpler subproblems, which are then solved recursively until convergence. The other fundamental observation is that the computational cost primarily depends on the efficiency of the linear step(s) (solver) of the algorithm that is directly related to the forward model.

\section{Modular formulation of reconstruction problem}

We now briefly explain how it is possible to decouple the physical aspects of the problem-the forward model that is specific to each modality - from the imposition of prior constraints on the signal, which is the part where advanced signal processing (e.g., compressed sensing and $\ell_{1}$-minimization) kicks in. For simplicity, we assume that the imaging model is linear. The reconstruction is then recast as the problem of estimating the signal, which is represented by the vector $x$, of size $N_{\text {rec }}$, from the "noisy" measurement vector $y=\mathrm{H} x+n$ of size $N_{\text {data }}$. Here, $\mathrm{H}$ is the system matrix of size $\left(N_{\text {data }} \times N_{\text {rec }}\right)$ that describes the physics of the imaging modality and $n$ is a disturbance or error term that typically represents measurement noise. In addition to the reconstruction problem being ill-posed (meaning that we cannot simply pseudo-invert $\mathrm{H}$ to retrieve $x$ from $y$ ), the main bootleneck relates to the size of the system matrix which is typically even too large to be stored within the computer.

To set the context and explain our vision, we focus on the class of algorithms summarized in Fig. 1, which is consistent with the state of the art in several modalities. Indeed, when we take $\mu \rightarrow \infty$, we have that $u=\mathrm{L} x$ so that 


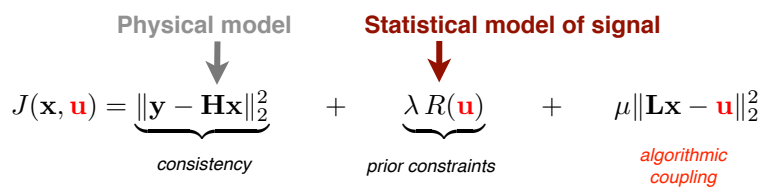

Schematic structure of reconstruction algorithm:
$N_{\text {iter }}\left[\begin{array}{rl}\text { Repeat } & \\ \mathbf{x}^{(n)}=\arg \min _{\mathbf{x}} J\left(\mathbf{x}, \mathbf{u}^{(n-1)}\right): & \text { Linear step (problem specific) } \\ \mathbf{u}^{(\mathbf{n})}=\arg \min _{\mathbf{u}} J\left(\mathbf{x}^{(n)}, \mathbf{u}\right): & \text { Statistical or "denoising" step } \\ \text { until stop criterion } & \end{array}\right.$

Fig. 1. Simplified structure of a variational image-reconstruction algorithm. The idea is to decouple the physical, modality-specific part of the problem from the signal-modeling aspects which is treated in a generic fashion.

we can identify the matrix $\mathrm{L}$ as a "sparsifying" transform such as the gradient or a wavelet decomposition. The prior constraints are imposed by taking $R(\cdot)$ to be a suitable penalty (or regularization) functional—the most popular choice being $R(u)=\|u\|_{1}$, which favors sparse solutions.

The conceptual advantage of this splitting is that the statistical step (ii) is completely generic (and therefore reusable), while the linear step (i) is application-specific with the advantage that one can reutilize existing linear reconstruction technology. This reasoning also suggests that one can essentially separate the issue of computational speed - the dominant cost being in the linear step-from the issue of reconstruction quality since the statistical modeling of the signal, if it is done properly, is responsible for the latter.

\section{Library structure}

Our inverse problem library, developed in MATLAB (MathWorks), relies on three main classes that are linear operators (LinOp), cost functions (Cost) and optimization algorithms (Opti). Specialized versions of these functions are available as subclasses (e.g. LinOpGrad, LinOpConv, CostL2, OptiGradDsct) to address (and exploit) the specifics of a variety of image reconstruction problems within the variational/Bayesian framework. This results in a modular toolbox for solving inverse problems that is powerful, flexible and easy to use, as illustrated in the following example.

Practical example Fig. 2 presents a library script that performs a 3D deconvolution with a total variation (TV) regularization. In effect, it solves the optimization problem,

$$
x_{\text {rec }}=\arg \min _{x \in \mathbb{R}^{N}} \frac{1}{2}\|\mathrm{H} x-y\|_{2}^{2}+\lambda\|x\|_{T V}+i_{\geqslant 0}(x),
$$

where $\mathrm{H}$ denotes the convolution operator, $\|\cdot\|_{T V}$ the TV "norm", $i_{\geqslant 0}(\cdot)$ the indicator function over positive vectors and $\lambda>0$ is a tradeoff factor between data fidelity and regularization. The two main steps in Fig. 2 consist respectively in building the elements (costs and operators) of the objective in (1) and defining the optimizer. Here, we use the ADMM algorithm [4] which is implemented to minimize functionals of the form:

$$
F(x):=F_{0}\left(\mathrm{H}_{0} x\right)+\sum_{n} F_{n}\left(\mathrm{H}_{n} x\right) .
$$

For the proposed 3D deconvolution example, we have $F_{0}=\frac{1}{2}\|\cdot-y\|_{2}^{2}, \mathrm{H}_{0}=\mathrm{H}, F_{1}=\lambda\|\cdot\|_{2,1}$ the mixed $\ell_{1}-\ell_{2}$ norm, $\mathrm{H}_{1}=\nabla$ the gradient operator, $F_{2}=i_{\geqslant 0}(\cdot)$ and $\mathrm{H}_{2}=\mathrm{I}_{\mathrm{N}}$ the identity operator.

As demonstration of the power of modularity, we can switch from a "standard" total variation to a more sophisticated Schatten-norm regularization [5] by simply substituting lines 8 and 9 by the code in Fig. 3. Similarly, one can readily adapt the presented script to another modality (new operator $\mathrm{H}$ ) by suitable modification of line 7 . Hence, one can apply the framework to different inverse problems and systematically test it out with various regularizers. With such a set-up, the imaging scientist can focus his/her effort on the implementation of $\mathrm{H}$ (or its adjoint=backprojection) - the only part of the code that is modality-specific.

Fig. 4 presents deconvolution results obtained using the two presented regularizations (TV and Hessian Shattennorm). One can observe that the Hessian-based regularization avoids the staircasing effect of TV. For both regularizations, the running time of the algorithm (150 iterations) is of the order of $10 \mathrm{~min}$ on a standard PC. 


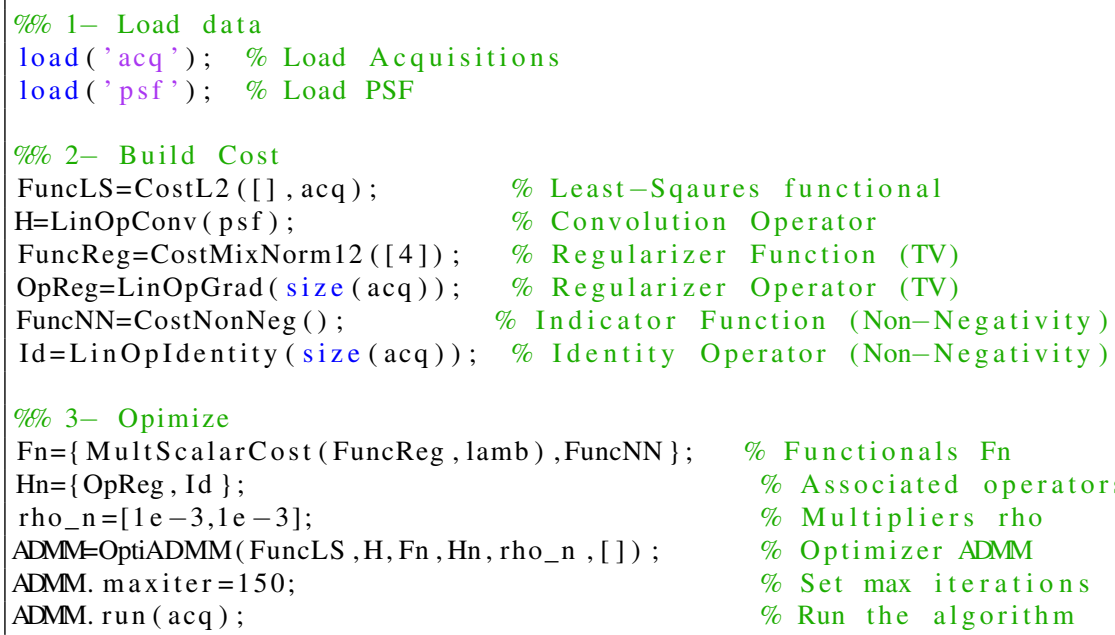

Fig. 2. Matlab script for 3D TV regularized deconvolution using the proposed library.

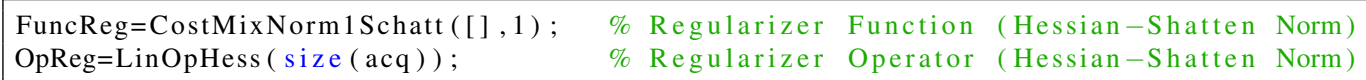

Fig. 3. Definition of the Hessian-Shatten norm regularizer (replaces lines 8 and 9 in Figure 2).
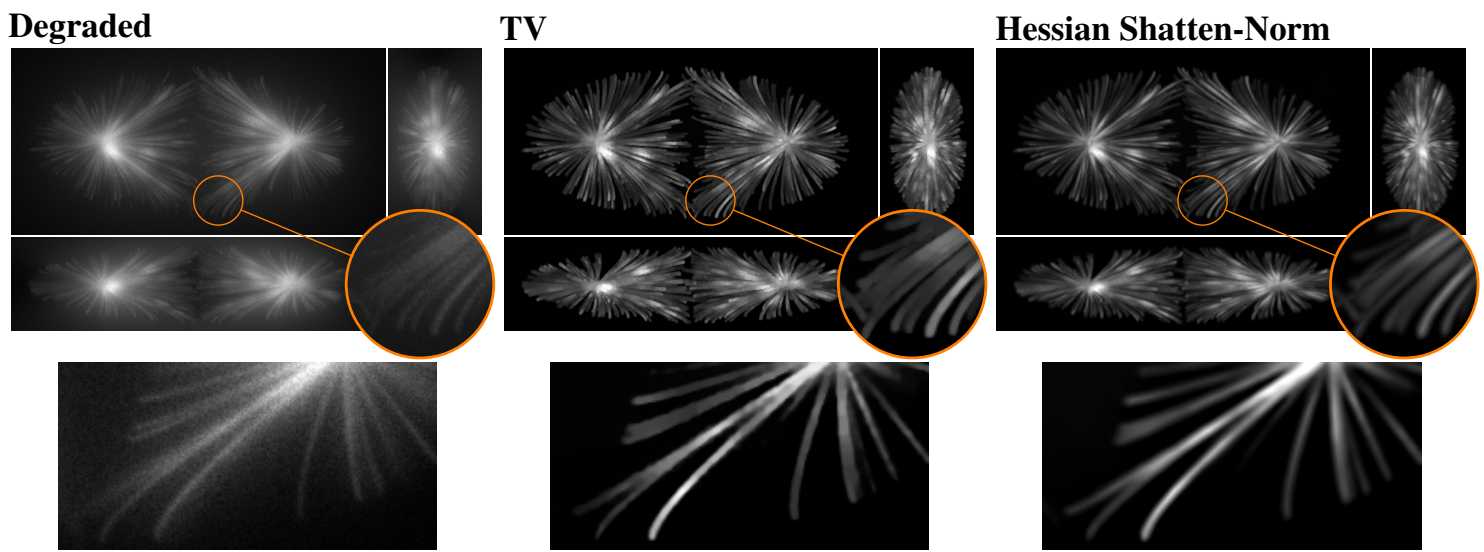

Fig. 4. Deconvolution results on the synthetic volume of size $256 \times 512 \times 128$ used in [1]. Top line: maximum intensity projection (MPI). Bottom line: Zoom on a XY slice.

\section{Acknowledgments}

This work was supported the European Research Council under Grant 692726 (H2020-ERC Project GlobalBioIm).

\section{References}

1. D. Sage, L. Donati, F. Soulez, D. Fortun, G. Schmit, A. Seitz, R. Guiet, C. Vonesch, and M. Unser, "DeconvolutionLab2: An open-source software for deconvolution microscopy," Methods-Image Processing for Biologists 115, 28-41 (2017).

2. M. Rostykus, F. Soulez, M. Unser, and C. Moser, “Compact lensless phase imager," Optics Express 25, 44384445 (2017).

3. U. S. Kamilov, I. N. Papadopoulos, M. H. Shoreh, A. Goy, C. Vonesch, M. Unser, and D. Psaltis, "A learning approach to optical tomography," Optica 2, 517-522 (2015).

4. S. Boyd, N. Parikh, E. Chu, and J. Eckstein, "Distributed optimization and statistical learning via the alternating direction method of multipliers," Information Systems Journal 3, 1-122 (2010).

5. S. Lefkimmiatis, J. Ward, and M. Unser, "Hessian Schatten-norm regularization for linear inverse problems," IEEE Transactions on Image Processing 22, 1873-1888 (2013). 